\title{
Prognostic factors associated with long-term mortality in 1445 patients with nontuberculous mycobacterial pulmonary disease: a 15-year follow-up study
}

\author{
Byung Woo Jhun (101,11, Seong Mi Moon ${ }^{2,11}$, Kyeongman Jeon (10) \\ 0 Jung Kwon ${ }^{1}$, Heejin Yoo ${ }^{3}$, Keumhee C. Carriere ${ }^{3,4}$, Hee Jae Huh ${ }^{5}$, \\ Nam Yong Lee ${ }^{5}$, Sung Jae Shin $\mathbb{1}^{6,7,8}$, Charles L. Daley ${ }^{9,10}$ and \\ Won-Jung Koh (1) ${ }^{1}$
}

@ERSpublications

The long-term mortality of patients with nontuberculous mycobacterial pulmonary disease was significantly associated with the aetiological organism, cavitary disease and certain demographic characteristics http://bit.ly/2kyXTHT

Cite this article as: Jhun BW, Moon SM, Jeon K, et al. Prognostic factors associated with long-term mortality in 1445 patients with nontuberculous mycobacterial pulmonary disease: a 15 -year follow-up study. Eur Respir J 2020; 55: 1900798 [https://doi.org/10.1183/13993003.00798-2019].

ABSTRACT Limited data are available regarding the prognostic factors for patients with nontuberculous mycobacterial pulmonary disease (NTM-PD). We investigated the prognostic factors associated with longterm mortality in NTM-PD patients after adjusting for individual confounders, including aetiological organism and radiological form.

A total of 1445 patients with treatment-naïve NTM-PD who were newly diagnosed between July 1997 and December 2013 were included. The aetiological organisms were as follows: Mycobacterium avium $(\mathrm{n}=655), M$. intracellulare $(\mathrm{n}=487), M$. abscessus $(\mathrm{n}=129)$ and $M$. massiliense $(\mathrm{n}=174)$. The factors associated with mortality in NTM-PD patients were analysed using a multivariable Cox model after adjusting for demographic, radiological and aetiological data.

The overall 5-, 10- and 15-year cumulative mortality rates for the NTM-PD patients were $12.4 \%, 24.0 \%$ and $36.4 \%$, respectively. On multivariable analysis, the following factors were significantly associated with mortality in NTM-PD patients: old age, male sex, low body mass index, chronic pulmonary aspergillosis, pulmonary or extrapulmonary malignancy, chronic heart or liver disease and erythrocyte sedimentation rate. The aetiological organism was also significantly associated with mortality: $M$. intracellulare had an adjusted hazard ratio (aHR) of 1.40, 95\% CI 1.03-1.91; M. abscessus had an aHR of 2.19, 95\% CI 1.36-3.51; and M. massiliense had an aHR of $0.99,95 \%$ CI $0.61-1.64$, compared to M. avium. Mortality was also significantly associated with the radiological form of NTM-PD for the cavitary nodular bronchiectatic form (aHR 1.70, 95\% CI 1.12-2.59) and the fibrocavitary form (aHR 2.12, 95\% CI 1.57-3.08), compared to the non-cavitary nodular bronchiectatic form.

Long-term mortality in patients with NTM-PD was significantly associated with the aetiological NTM organism, cavitary disease and certain demographic characteristics.

This article has supplementary material available from erj.ersjournals.com

This study is registered with ClinicalTrials.gov identifier: NCT00970801.

Received: 20 April 2019 | Accepted after revision: 18 Sept 2019

Copyright OERS 2020 


\section{Introduction}

The burden of nontuberculous mycobacterial pulmonary disease (NTM-PD) is rapidly increasing worldwide [1, 2]. Although the major causative organisms of NTM-PD differ by geographical region, the most common pathogens include Mycobacterium avium complex (MAC), which includes M. avium and M. intracellulare; and M. abscessus (MAB), which includes M. abscessus subsp. abscessus (hereafter referred to as M. abscessus) and M. abscessus subsp. massiliense (hereafter referred to as M. massiliense) $[1,2]$. NTM-PD has traditionally been divided into two clinical phenotypes: the fibrocavitary form, which is characterised by cavitary lesions typically located in the upper lobes; and the nodular bronchiectatic (NB) form, which presents as bilateral bronchiectasis with nodular infiltrates involving the middle lung zones $[3,4]$. However, some patients with the NB form have cavitary lesions [5, 6].

NTM-PD has substantial heterogeneity with regard to disease progression, with some patients showing rapid progression and others showing stable disease, especially in cases of the NB form [7-11]. The current guidelines state that a diagnosis of NTM-PD alone does not necessitate immediate commencement of treatment and that the decision should be made based on the potential risks and benefits of therapy for individual patients $[3,4]$. In particular, currently recommended multidrug regimens are not well tolerated by elderly or frail individuals with comorbidities and show unsatisfactory treatment efficacy despite long-term therapy [12-15]. Furthermore, repeated reinfection by different strains or species frequently occurs during and after antibiotic treatment $[6,16-18]$. These conditions make treating NTM-PD challenging. Therefore, it is crucial to identify reliable prognostic factors to determine the optimal treatment strategies.

Previous studies evaluating the prognosis of NTM-PD described varying rates of mortality and reported some demographic or clinico-radiological characteristics as prognostic factors [19-22]. However, the data have frequently shown discrepancies, some of which may be due to small numbers of subjects, lack of detailed clinical or microbiological profiles and/or limited study periods. Therefore, few studies have addressed the long-term prognosis for NTM-PD through analysis of detailed clinical data. This 15-year follow-up study was performed to evaluate the long-term prognosis for NTM-PD and the factors associated with mortality in 1445 patients with NTM-PD, after adjusting for individual confounders including aetiological organism and radiological form.

\section{Methods}

\section{Study population}

A total of 1603 consecutive patients newly diagnosed with MAC- or MAB-PD between July 1997 and December 2013 were identified from the NTM Registry of Samsung Medical Center, a 1979-bed referral hospital in Seoul, South Korea. From July 1997 to December 2007, data were obtained from a retrospective cohort, and beginning in January 2008, data were obtained from an ongoing Institutional Review Board-approved prospective observational cohort (ClinicalTrials.gov identifier: NCT00970801) [6, 18, 23, 24]. Patients were excluded from the study if they had an unclassifiable form of NTM-PD ( $\mathrm{n}=95)$, such as a solitary pulmonary nodule that could not be categorised as either the fibrocavitary or NB form on chest radiograph or high-resolution computed tomography; MAB-PD without further species identification $(\mathrm{n}=51) ;$ M. bolletii disease $(\mathrm{n}=2)$; or an unavailable death certificate due to emigration $(\mathrm{n}=10)$. Finally, 1445 patients diagnosed with treatment-naïve NTM-PD were included in this study (figure 1). All patients met the diagnostic criteria for NTM-PD $[3,25]$.

Patient follow-up data were last updated on 30 June 2017. All dates of patient deaths were ascertained from the medical records or the database of the National Health Insurance Service.

Microbiological and radiological examinations

Acid-fast bacilli smears and cultures of lower respiratory tract samples were obtained using standard methods. NTM species were identified using polymerase chain reaction-restriction fragment length

Affiliations: ${ }^{1}$ Division of Pulmonary and Critical Care Medicine, Dept of Medicine, Samsung Medical Center, Sungkyunkwan University School of Medicine, Seoul, South Korea. ${ }^{2}$ Division of Pulmonary and Critical Care Medicine, Dept of Medicine, Samsung Changwon Hospital, Sungkyunkwan University School of Medicine, Changwon, South Korea. ${ }^{3}$ Statistics and Data Center, Samsung Medical Center, Seoul, South Korea. ${ }^{4}$ Dept of Mathematical and Statistical Sciences, University of Alberta, Edmonton, AB, Canada. ${ }^{5}$ Dept of Laboratory Medicine and Genetics, Samsung Medical Center, Sungkyunkwan University School of Medicine, Seoul, South Korea. ${ }^{6}$ Dept of Microbiology, Yonsei University College of Medicine, Seoul, South Korea. ${ }^{7}$ Institute for Immunology and Immunological Disease, Yonsei University College of Medicine, Seoul, South Korea. ${ }^{8}$ Brain Korea 21 PLUS Project for Medical Science, Yonsei University College of Medicine, Seoul, South Korea. ${ }^{9}$ Division of Mycobacterial and Respiratory Infections, Dept of Medicine, National Jewish Health, Denver, CO, USA. ${ }^{10}$ Dept of Medicine, University of Colorado, Aurora, CO, USA. ${ }^{11}$ Both authors contributed equally.

Correspondence: Byung Woo Jhun, Samsung Medical Center, Division of Pulmonary and Critical Care Medicine, Irwon-ro 81, Gangnam-gu, Seoul, 06351, South Korea. E-mail: byungwoo.jhundagmail.com 


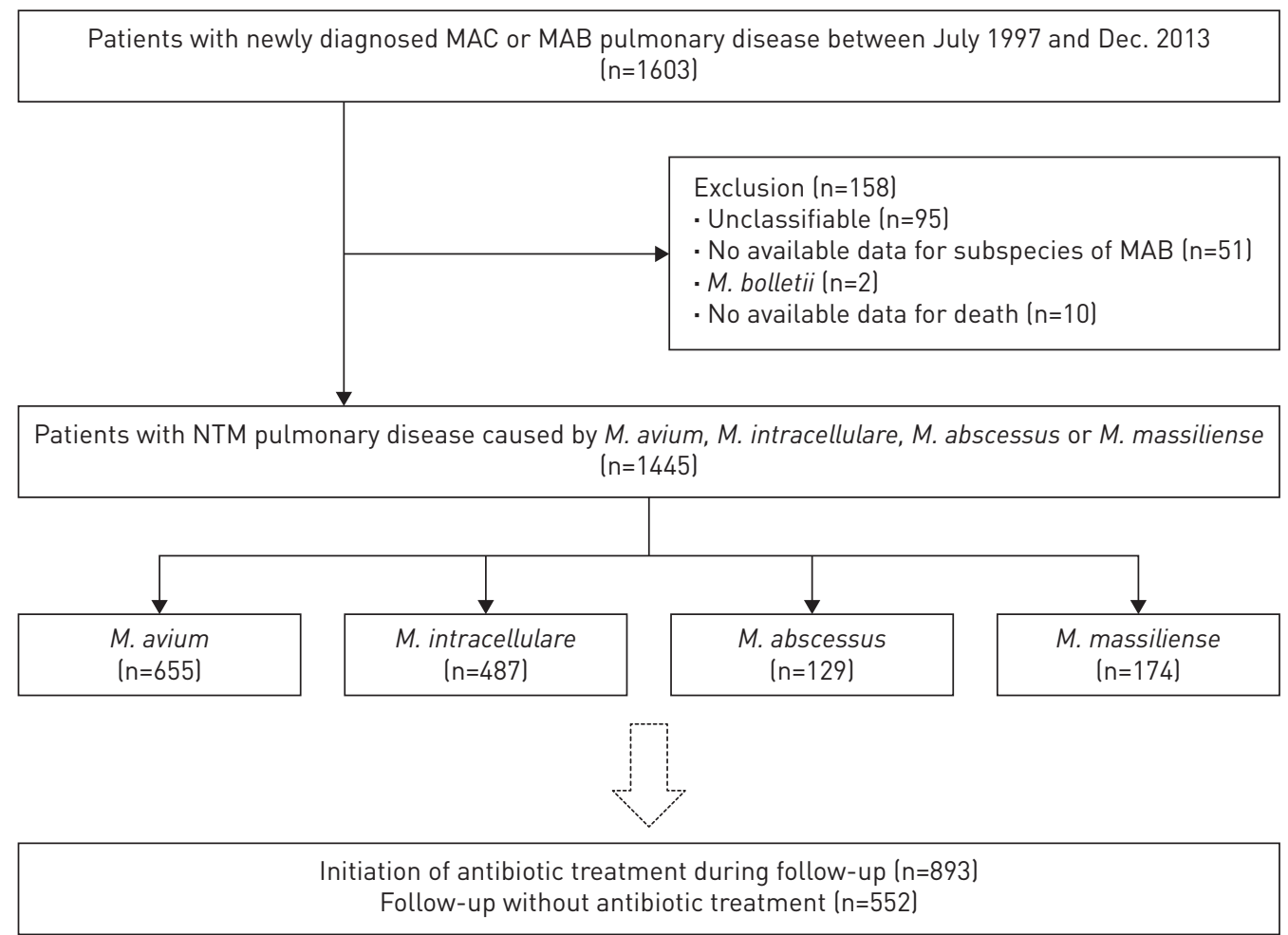

FIGURE 1 Study patients. MAC: Mycobacterium avium complex; MAB: M. abscessus; NTM: nontuberculous mycobacterial.

polymorphism analysis of the $r p o B$ gene or reverse-blot hybridisation assay of $r p o B[6,18,23,24]$. The radiological form was categorised as fibrocavitary, cavitary NB or non-cavitary NB form, as described previously $[6,18,23,24]$.

\section{Patient management and treatment modalities}

After the diagnosis of NTM-PD was confirmed, antibiotic therapy was initiated at the discretion of the attending physician, with consideration of the potential risks and benefits. In NTM-PD patients presenting with cavitary lesions, including the fibrocavitary and cavitary NB forms, antibiotic therapy was usually initiated after diagnosis of NTM-PD. In patients presenting with the non-cavitary NB form, bronchiectasis was initially managed using airway clearance measures and short-term antibiotic therapy against acute exacerbation of bronchiectasis. If symptoms and radiological abnormalities were not improved after bronchiectasis management, the attending physician would start antibiotic therapy for NTM-PD [6, 18, 23, 24].

Multidrug antibiotic therapy was applied for treatment of NTM-PD according to the aetiological organism. Briefly, in MAC-PD patients, an oral macrolide-based regimen that included ethambutol and rifampin was used. Aminoglycosides were additionally administered in patients with severe disease for the first several months $[6,23]$. In MAB-PD patients, an initial 2- to 4-week course of intravenous amikacin and imipenem (or cefoxitin) was administered during hospitalisation, together with oral medications including a macrolide always given plus either fluoroquinolone or doxycycline. After discharge, oral regimens were maintained $[18,24]$. Some patients with refractory NTM-PD received oral clofazimine or inhaled amikacin [26, 27].

\section{Statistical analyses}

Data are presented as number (\%) for categorical variables and median (interquartile range (IQR)) for continuous variables. Preliminary analysis compared the outcome based on these categories using ANOVA or the chi-square test. The Kaplan-Meier method was used to estimate the cumulative survival rates of NTM-PD patients, and the log-rank test was used to compare survival curves. Selection of risk factors for mortality was conducted based on the multivariable Cox proportional-hazards regression model, via the least absolute shrinkage and selection operator selection (LASSO) method, considering all clinical and other variables. The LASSO variable selection technique was applied because it is useful when there are many covariates relative to the sample size, and this method aided in assessing the relative importance of 
their effects on the outcome to encourage a simple parsimonious model [28]. The treatment status (initiation of antibiotic therapy or not) was included in the Cox model as a time-dependent explanatory variable during the follow-up. We verified the assumptions of the Cox proportional-hazards model using the Schoenfeld residuals and measured the predictive accuracy of the model using Harrell's c-index. Finally, a clinically and statistically viable final model was identified. Statistical analyses were performed using SAS (version 9.4; SAS Institute Inc., Cary, NC, USA) and R software (version 3.4.3; R Development Core Team, Vienna, Austria). Two-sided p-values $<0.05$ were considered statistically significant.

\section{Results}

\section{Baseline characteristics}

The baseline characteristics of 1445 patients at the time of diagnosis with NTM-PD are shown in table 1. In total, 509 (35\%) patients were aged 65 years or older, 558 (39\%) patients were male, 27\% were smokers and $22 \%$ had a low body mass index $\left(<18.5 \mathrm{~kg} \cdot \mathrm{m}^{-2}\right)$. The most common underlying respiratory disease was previous tuberculosis $(n=566,39 \%)$, followed by chronic obstructive pulmonary disease or bronchial asthma $(n=147,10 \%)$, pulmonary malignancy $(n=45,3 \%)$ and chronic pulmonary aspergillosis $(n=31$, $2 \%)$. The median (IQR) erythrocyte sedimentation rate (ESR) was $29 \mathrm{~mm} \cdot \mathrm{h}^{-1}\left(16-53 \mathrm{~mm} \cdot \mathrm{h}^{-1}\right)$. Positive sputum acid-fast bacilli smears were identified in 701 (49\%) patients, and M. avium ( $\mathrm{n}=655,45 \%)$ was the most common aetiological organism, followed by $M$. intracellulare $(\mathrm{n}=487,34 \%)$; M. massiliense $(\mathrm{n}=174$, $12 \%)$; and M. abscessus ( $\mathrm{n}=129,9 \%)$. Of the study patients, 1044 (72\%) had non-cavitary NB, $143(10 \%)$ had cavitary NB and 258 (18\%) had fibrocavitary forms.

\section{Follow-up and overall mortality}

The median (IQR) follow-up period for all 1445 patients with NTM-PD was 77.6 months (55.3-112.4 months). Additionally, 893 (62\%) started antibiotic therapy at a median (IQR) of 6.1 months (1.5-21.7 months) after diagnosis of NTM-PD, whereas the remaining 552 (38\%) did not receive antibiotic therapy during their median (IQR) follow-up period of 78.9 months (54.9-112.4 months) after diagnosis of NTM-PD. Regarding the NTM aetiology, patients with $M$. intracellulare (70\%, 342 out of 487) or M. massiliense (69\%, 120 out of 174) were more likely to start antibiotic therapy than were those with M. avium (55\%, 361 out of 655 ) or M. abscessus

\section{TABLE 1 Baseline characteristics of all study patients at diagnosis ( $n=1445$ )}

\begin{tabular}{|c|c|}
\hline Age years & $60(51-68)$ \\
\hline Age $\geqslant 65$ years & 509 (35) \\
\hline Male sex & 558 (39) \\
\hline Ex- or current smoker & $383(27)$ \\
\hline Body mass index $<18.5 \mathrm{~kg} \cdot \mathrm{m}^{-2}$ & $312(22)$ \\
\hline \multicolumn{2}{|l|}{ Underlying disease } \\
\hline Previous pulmonary tuberculosis & 566 (39) \\
\hline COPD or bronchial asthma & $147(10)$ \\
\hline Chronic pulmonary aspergillosis & $31(2)$ \\
\hline Pulmonary malignancy & $45(3)$ \\
\hline Extrapulmonary malignancy & $208(14)$ \\
\hline Diabetes mellitus & $101(7)$ \\
\hline Chronic heart disease & $136(9)$ \\
\hline Chronic liver disease & $104(7)$ \\
\hline \multicolumn{2}{|l|}{ Symptom } \\
\hline Cough & $1028(71)$ \\
\hline Sputum & $1064(74)$ \\
\hline Haemoptysis & 324 (22) \\
\hline Erythrocyte sedimentation rate $\mathrm{e}^{\#} \mathrm{~mm} \cdot \mathrm{h}^{-1}$ & $29(16-53)$ \\
\hline Sputum smear positivity & 701 (49) \\
\hline \multicolumn{2}{|l|}{ Aetiology } \\
\hline M. avium & $655(45)$ \\
\hline M. intracellulare & $487(34)$ \\
\hline M. abscessus & $129(9)$ \\
\hline M. massiliense & $174(12)$ \\
\hline \multicolumn{2}{|l|}{ Radiological form } \\
\hline Non-cavitary nodular bronchiectatic & 1044 (72) \\
\hline Cavitary nodular bronchiectatic & $143(10)$ \\
\hline Fibrocavitary & $258(18)$ \\
\hline
\end{tabular}

Data are presented as median (interquartile range) or $\mathrm{n}(\%){ }^{\#}: \mathrm{n}=1181$ due to missing data. 
(54\%, 70 out of 129) $(\mathrm{p}<0.001)$. In terms of the radiological form of the disease, patients with the fibrocavitary form were more likely to start antibiotic therapy $(86 \%, 223$ out of 258$)$ than were those with the cavitary NB $(84 \%, 120$ out of 143$)$ or non-cavitary NB forms $(53 \%, 550$ out of 1044$)(p<0.001)$.

The overall 5-, 10- and 15-year cumulative mortality rates for all 1445 patients were $12.4 \%, 24.0 \%$ and $36.4 \%$, respectively. Over the 15-year follow-up period, $283(20 \%)$ of the study patients died. Mortality rates did not differ between the treated and untreated groups (supplementary figure S1).

\section{Survival analysis according to NTM aetiology}

We evaluated the cumulative survival rate of the patients according to the four aetiological organisms of NTM-PD included in this study (figure 2a). Overall, the survival rates were higher in patients with M. massiliense or M. avium compared to those with M. abscessus or M. intracellulare (Kaplan-Meier analysis, log-rank test $\mathrm{p}<0.001$ ). The overall 5-, 10- and 15-year cumulative mortality rates for patients with M. massiliense disease were $7.3 \%, 18.1 \%$ and $23.5 \%$, respectively, which were similar to those with M. avium disease $(8.0 \%, 17.2 \%$ and $27.9 \%$, respectively). The overall 5-, 10 - and 15 -year cumulative mortality rates for patients with $M$. intracellulare disease were $20.3 \%, 33.6 \%$ and $45.5 \%$, respectively. The overall 5-, $10-$ and 15-year cumulative mortality rates for patients with M. abscessus disease were $11.4 \%, 29.8 \%$ and $50.6 \%$, respectively. The log-rank test indicated that the differences in the survival curves between both the $M$. massiliense versus $M$. intracellulare groups and the $M$. avium versus $M$. intracellulare groups were significant $(\mathrm{p}<0.001$ for both). Additionally, differences in characteristics among study patients according to NTM aetiology are shown in supplementary table S1. Mortality rates according to treatment initiation for the different aetiological organisms did not differ significantly (supplementary figure S2).

\section{Survival analysis according to radiological form}

We also evaluated the cumulative survival rate according to the radiological form of NTM-PD in the study patients (figure $2 \mathrm{~b}$ ). Overall, the survival rates were higher in patients with the non-cavitary NB form compared to those with cavitary disease, including the cavitary NB and fibrocavitary forms (Kaplan-Meier analysis, log-rank test $\mathrm{p}<0.001)$. The overall 5-, 10 - and 15-year cumulative mortality rates for the non-cavitary NB form were $6.3 \%, 16.0 \%$ and $26.6 \%$, respectively. Patients with the fibrocavitary form consistently had the highest mortality rates over the entire follow-up period, with overall 5-, 10- and 15-year cumulative mortality rates of $35.1 \%, 47.0 \%$ and $65.4 \%$, respectively. Patients with the cavitary NB form had mortality rates intermediate between those with the non-cavitary NB and fibrocavitary forms. The log-rank test indicated that the pairwise differences in survival curves among the three groups were all significant (all $\mathrm{p}<0.001)$. Additionally, differences in characteristics among study patients according to radiological form are shown in supplementary table S2. Mortality rates according to treatment initiation for the different radiological forms did not differ significantly (supplementary figure S3).

a)

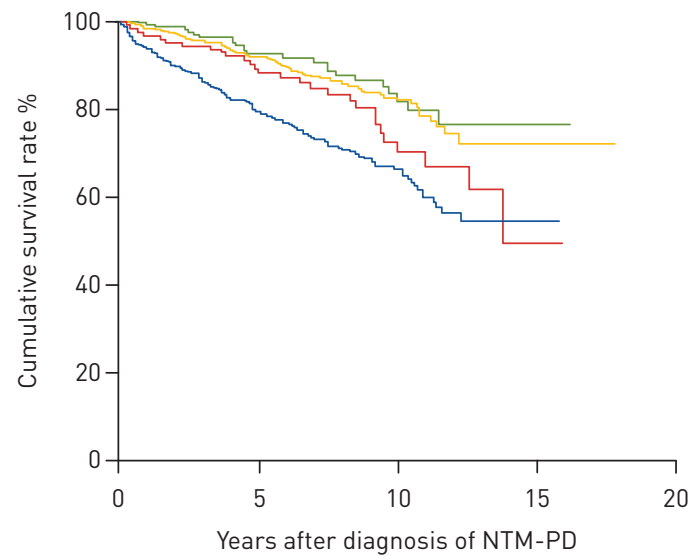

b)

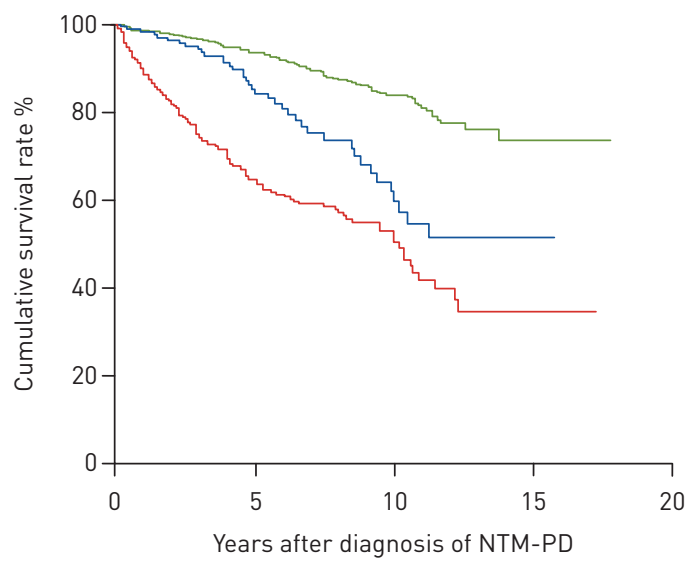

Number at risk (mortality \%)

- M. massiliense $174(0.0)$

- M. avium $655(0.0)$

- M. intracellulare $487(0.0)$

- M. abscessus
$136(7.3)$

$481(8.0)$

$318(20.3)$

$96(11.4)$
$48(18.1)$

$146(17.2)$

$94(33.6)$

$30(29.8)$
23 (23.5)

31 (27.9)

$28(45.5)$

$4(50.6)$
FIGURE 2 a) Cumulative survival rates of patients with nontuberculous mycobacterial pulmonary disease (NTM-PD) according to aetiological organism. b) Cumulative survival rates of patients with NTM-PD according to radiological form. NB: nodular bronchiectatic. 
Prognostic factors of mortality in NTM-PD

The prognostic factors associated with all-cause mortality were explored (table 2). In univariable analysis, the factors that appeared to be significantly associated with elevated risk of mortality in NTM-PD included old age $\left(\geqslant 65\right.$ years); male sex; smoking; low body mass index $\left(<18.5 \mathrm{~kg} \cdot \mathrm{m}^{-2}\right)$; various underlying diseases; respiratory symptoms; ESR; sputum smear positivity; $M$. intracellulare disease (reference: M. avium); cavitary disease, including the cavitary NB and fibrocavitary forms (reference: non-cavitary NB); and initiation of treatment.

In multivariable adjustment, the LASSO method indicated that old age ( $\geqslant 65$ years), male sex, low body mass index $\left(<18.5 \mathrm{~kg} \cdot \mathrm{m}^{-2}\right)$, underlying diseases including chronic pulmonary aspergillosis, malignancy, chronic heart or chronic liver disease and ESR were significantly associated with mortality in NTM-PD. In addition, NTM aetiology (reference: $M$. avium) was also significantly associated with mortality: M. intracellulare had an adjusted hazard ratio (aHR) of 1.40, 95\% CI 1.03-1.91; M. abscessus, aHR 2.19, 95\% CI 1.36-3.51; and M. massiliense, aHR 0.99, 95\% CI 0.61-1.64. Mortality was also significantly associated with radiological form, especially in cavitary disease (reference: non-cavitary NB): cavitary NB form, aHR 1.70, 95\% CI 1.12-2.59; and fibrocavitary form, aHR 2.20, 95\% CI 1.57-3.08.

\section{Discussion}

To our knowledge, this is the largest study to date that evaluates the long-term mortality and the prognostic factors associated with NTM-PD caused by either of the major pathogens MAC or MAB, after adjustment for numerous individual confounders. The survival rate of patients with NTM-PD was significantly influenced

TABLE 2 Analysis of prognostic factors related to mortality in patients with nontuberculous mycobacterial pulmonary disease

\begin{tabular}{|c|c|c|c|c|}
\hline \multirow[t]{2}{*}{ Characteristics } & \multicolumn{2}{|c|}{ Univariable } & \multicolumn{2}{|c|}{ Final multivariable } \\
\hline & HR $(95 \%$ CI $)$ & p-value & $\operatorname{HR}(95 \% \mathrm{CI})$ & p-value \\
\hline Age $\geqslant 65$ years & $4.36(3.40-5.58)$ & $<0.001$ & $3.01(2.23-4.06)$ & $<0.001$ \\
\hline Male sex & $3.45(2.70-4.40)$ & $<0.001$ & $2.68(2.01-3.57)$ & $<0.001$ \\
\hline Ex- or current smoker & $2.67(2.11-3.38)$ & $<0.001$ & & \\
\hline $\begin{array}{l}\text { Body mass index }<18.5 \mathrm{~kg} \cdot \mathrm{m}^{-2} \\
\text { Underlying disease }\end{array}$ & $3.85(3.05-4.86)$ & $<0.001$ & $1.96(1.50-2.56)$ & $<0.001$ \\
\hline Previous pulmonary tuberculosis & $1.98(1.57-2.51)$ & $<0.001$ & & \\
\hline COPD or bronchial asthma & $2.07(1.53-2.80)$ & $<0.001$ & & \\
\hline Chronic pulmonary aspergillosis & $3.49(2.07-5.88)$ & $<0.001$ & $1.77(1.01-3.11)$ & 0.047 \\
\hline Pulmonary malignancy & $6.69(4.57-9.80)$ & $<0.001$ & $2.65(1.69-4.16)$ & $<0.001$ \\
\hline Extra-pulmonary malignancy & $1.98(1.50-2.61)$ & $<0.001$ & $1.61(1.15-2.26)$ & 0.006 \\
\hline Diabetes mellitus & $2.46(1.77-3.43)$ & $<0.001$ & & \\
\hline Chronic heart disease & $2.26(1.65-3.09)$ & $<0.001$ & $2.50(1.73-3.61)$ & $<0.001$ \\
\hline Chronic liver disease & $1.78(1.23-2.55)$ & 0.002 & $1.86(1.24-2.78)$ & 0.003 \\
\hline \multicolumn{5}{|l|}{ Symptom } \\
\hline Cough & $1.42(1.06-1.90)$ & 0.019 & & \\
\hline Sputum & $1.49(1.10-2.03)$ & 0.010 & & \\
\hline Haemoptysis & $0.73(0.55-0.98)$ & 0.036 & & \\
\hline Erythrocyte sedimentation rate ${ }^{\#} \mathrm{~mm} \cdot \mathrm{h}^{-1}$ & $1.03(1.02-1.03)$ & $<0.001$ & $1.02(1.01-1.02)$ & $<0.001$ \\
\hline Sputum smear positivity & $2.52(1.96-3.24)$ & $<0.001$ & & \\
\hline \multicolumn{5}{|l|}{ Aetiology } \\
\hline M. avium & Reference & & Reference & \\
\hline M. intracellulare & $2.29(1.76-2.98)$ & $<0.001$ & $1.40(1.03-1.91)$ & 0.034 \\
\hline M. abscessus & $1.47(0.96-2.25)$ & 0.076 & $2.19(1.36-3.51)$ & 0.001 \\
\hline M. massiliense & $0.86(0.55-1.36)$ & 0.525 & $0.99(0.61-1.64)$ & 0.990 \\
\hline \multicolumn{5}{|l|}{ Radiological form } \\
\hline Non-cavitary nodular bronchiectatic & Reference & & Reference & \\
\hline Cavitary nodular bronchiectatic & $2.45(1.70-3.52)$ & $<0.001$ & $1.70(1.12-2.59)$ & 0.013 \\
\hline Fibrocavitary & $4.74(3.69-6.09)$ & $<0.001$ & $2.20(1.57-3.08)$ & $<0.001$ \\
\hline Treatment initiation & $1.86(1.44-2.39)$ & $<0.001$ & $1.32(0.97-1.80)$ & 0.077 \\
\hline
\end{tabular}

The assumption of proportional hazard was deemed satisfied with the Schoenfeld residual correlation with time $<0.15$ for all covariates. Overall, the model's predictive accuracy was indicated excellent, with a c-index of 0.86 (95\% Cl 0.82-0.90). HR: hazard ratio. ${ }^{\#}: \mathrm{n}=1181$ due to missing data. 
by the aetiological organism, cavitary disease and certain demographic characteristics. In particular, the mortality rates of patients with either M. intracellulare or M. abscessus and cavitary disease were high.

As the burden of NTM-PD is increasing worldwide, studies on mortality in patients with NTM-PD have been reported in many regions [29, 30], including Japan [5, 20, 31], Europe [19, 32] and North America [21, 22, 33-35]. A study in Japan showed overall 5- and 10-year mortality rates for MAC-PD of $23.9 \%$ and $46.5 \%$, respectively, which were similar to, although slightly higher than, those of our patients with $M$. intracellulare disease [20]. However, the Japanese study did not distinguish between $M$. intracellulare and M. avium, and its cohort had a higher rate of comorbidities. A study conducted in Denmark reported a 5 -year mortality rate of $40.1 \%$ among NTM-PD patients, with the aetiological organisms including MAC, $\mathrm{MAB}$ and other species [19]. The major difference between the Danish study and our study was that most of its definite cases of NTM-PD had progressive disease requiring treatment, and the authors also did not test for the presence of cavitary disease. In addition, a recent study in the USA that evaluated 106 patients with NTM-PD caused by various NTM species reported a mortality rate of 25\% during a 5-year follow-up [22]. These discrepant results suggest that the prognosis of NTM-PD may differ depending on the clinical or demographic characteristics of the cohort or on the stage of disease at the time of diagnosis, and indicate that evaluation of prognostic factors can be more informative than only assessing mortality.

One of the most notable findings of the present study was the difference in mortality according to NTM aetiology, with a poor prognosis for patients with disease caused by $M$. intracellulare or $M$. abscessus. Globally, MAC and MAB are the major aetiological organisms of NTM-PD, and studies suggest that there is inter- and intra-species heterogeneity with regard to NTM virulence and influence on disease severity. For example, $M$. intracellulare disease had a more severe clinical presentation and poorer treatment outcomes when compared with M. avium disease in one study [36]. Additionally, compared with M. abscessus disease, M. massiliense disease responds more favourably to macrolide-containing antibiotic therapy $[18,37,38]$. In this context, our data clearly showed that the accurate identification of the species or subspecies of the aetiological NTM organism is very important in the prognosis of NTM-PD.

Another important finding of the present study was that survival rates of NTM-PD differed according to the presence of cavitary lesions, even in the NB form. Traditionally, the fibrocavitary form is regarded to have a poorer prognosis than NB disease does [3, 4]. Previous studies from Japan [20] and the USA [22] showed that the fibrocavitary form was an important prognostic factor of poor outcomes, and in those studies, the disease phenotype was categorised as either the fibrocavitary or NB form. Regarding the presence of cavitary lesions in some patients with NB NTM-PD, however, a recent study suggested that the radiological findings of NTM-PD could be classified into three phenotypes (i.e., fibrocavitary, cavitary NB and non-cavitary NB forms), and in MAC-PD, these phenotypes have different outcomes after antibiotic treatment [6]. The present study suggested that the presence of cavities was also an important prognostic factor in predicting mortality, even among patients with NB NTM-PD. One may assume that the untreated NB form without cavitary lesions may progress to cavitary NB or more diffuse disease [39, 40]. Therefore, the detection of cavitary lesion(s) on computed tomography scans would be important for predicting treatment outcomes and mortality in patients with NTM-PD.

In our analysis, several demographic characteristics similar to those described previously were identified as prognostic factors. Typically, factors such as old age, male sex, comorbidities, concurrent malignancy and inflammatory markers were reported to be associated with poor prognosis $[5,19,20,31]$. However, very few studies included "treatment-related risk factors" in survival analysis [5, 20]. In our multivariable analysis, treatment initiation was not significantly associated with prognosis. Most of the patients who did not receive treatment had mild disease and did not require treatment, whereas those who started treatment had advanced disease and did require treatment. Therefore, treatment initiation may have contributed to improving the survival rate in advanced NTM-PD patients, bringing the rate closer to that for untreated patients, which may explain the above results. A similar phenomenon was observed in a previous study that compared survival rates among colonised, possible and definite NTM disease [19]. In that study, the difference in survival rate between the three groups was small, with the authors proposing that this resulted from most patients with definite NTM being treated according to the accepted guidelines.

There are some limitations to our study. First, because it was not a population-based cohort study and was conducted at a single referral centre, our data may not be generalisable to other geographic areas. Second, we only evaluated all-cause mortality, not NTM-PD-specific mortality, due to a lack of detailed information. Third, we only included patients with NTM-PD caused by the four major pathogens of MAC and MAB. Lastly, the effects of treatment regimens on survival rate were not evaluated, mainly due to differences in regimens between MAC- and MAB-PD.

In conclusion, long-term mortality in patients with NTM-PD was significantly associated with the aetiological organism of NTM, cavitary disease and certain demographic characteristics. 
Author contributions: Study conception and design: B.W. Jhun, S.M. Moon and W-J. Koh. Data acquisition and analysis: B.W. Jhun, S.M. Moon, K. Jeon, O.J. Kwon, H. Yoo, K.C. Carriere, H.J. Huh, N.Y. Lee, S.J. Shin and W-J. Koh. Data interpretation and manuscript writing: B.W. Jhun, S.M. Moon and W-J. Koh. Critical revision and final approval of the manuscript: B.W. Jhun, S.M. Moon, K. Jeon, O.J. Kwon, H. Yoo, K.C. Carriere, H.J. Huh, N.Y. Lee, S.J. Shin, C.L. Daley and W-J. Koh.

Conflict of interest: B.W. Jhun has nothing to disclose. S.M. Moon has nothing to disclose. K. Jeon has nothing to disclose. O.J. Kwon has nothing to disclose. H. Yoo has nothing to disclose. K.C. Carriere has nothing to disclose. H.J. Huh has nothing to disclose. N.Y. Lee has nothing to disclose. S.J. Shin has nothing to disclose. C.L. Daley reports grants from Insmed Inc., and has served on advisory boards for Insmed, Johnson \& Johnson, Spero and Horizon, outside the submitted work. W-J. Koh reports personal fees for consultancy from Insmed, outside the submitted work.

Support statement: This work was supported by the National Research Foundation of Korea (NRF) funded by the Korea government (MSIT) (NRF-2018R1A2A1A05018309). The sponsor had no role in the design of the study, the collection and analysis of the data, or the preparation of the manuscript. Funding information for this article has been deposited with the Crossref Funder Registry.

\section{References}

1 Prevots DR, Marras TK. Epidemiology of human pulmonary infection with nontuberculous mycobacteria: a review. Clin Chest Med 2015; 36: 13-34. doi:10.1016/j.ccm.2014.10.002

2 Adjemian J, Daniel-Wayman S, Ricotta E, et al. Epidemiology of nontuberculous mycobacteriosis. Semin Respir Crit Care Med 2018; 39: 325-335. doi:10.1055/s-0038-1651491

3 Griffith DE, Aksamit T, Brown-Elliott BA, et al. An official ATS/IDSA statement: diagnosis, treatment, and prevention of nontuberculous mycobacterial diseases. Am J Respir Crit Care Med 2007; 175: 367-416. doi:10.1164/ rccm.200604-571ST

4 Haworth CS, Banks J, Capstick T, et al. British Thoracic Society guidelines for the management of non-tuberculous mycobacterial pulmonary disease (NTM-PD). Thorax 2017; 72: Suppl. 2, ii1-ii64. doi:10.1136/ thoraxjnl-2017-210927

5 Gochi M, Takayanagi N, Kanauchi T, et al. Retrospective study of the predictors of mortality and radiographic deterioration in 782 patients with nodular/bronchiectatic Mycobacterium avium complex lung disease. BMJ Open 2015; 5: e008058. doi:10.1136/bmjopen-2015-008058

6 Koh WJ, Moon SM, Kim SY, et al. Outcomes of Mycobacterium avium complex lung disease based on clinical phenotype. Eur Respir J 2017; 50: 1602503. doi:10.1183/13993003.02503-2016

7 Hwang JA, Kim S, Jo KW, et al. Natural history of Mycobacterium avium complex lung disease in untreated patients with stable course. Eur Respir J 2017; 49: 1600537. doi:10.1183/13993003.00537-2016

8 Park TY, Chong S, Jung JW, et al. Natural course of the nodular bronchiectatic form of Mycobacterium avium complex lung disease: long-term radiologic change without treatment. PLoS One 2017; 12: e0185774.

9 Henkle E, Novosad SA, Shafer S, et al. Long-term outcomes in a population-based cohort with respiratory nontuberculous mycobacteria isolation. Ann Am Thorac Soc 2017; 14: 1120-1128. doi:10.1513/ AnnalsATS.201610-801OC

10 Kwon BS, Lee JH, Koh Y, et al. The natural history of non-cavitary nodular bronchiectatic Mycobacterium avium complex lung disease. Respir Med 2019; 150: 45-50. doi:10.1016/j.rmed.2019.02.007

11 Moon SM, Jhun BW, Baek SY, et al. Long-term natural history of non-cavitary nodular bronchiectatic nontuberculous mycobacterial pulmonary disease. Respir Med 2019; 151: 1-7. doi:10.1016/j.rmed.2019.03.014

12 Kwak N, Park J, Kim E, et al. Treatment outcomes of Mycobacterium avium complex lung disease: a systematic review and meta-analysis. Clin Infect Dis 2017; 65: 1077-1084. doi:10.1093/cid/cix517

13 Diel R, Nienhaus A, Ringshausen FC, et al. Microbiologic outcome of interventions against Mycobacterium avium complex pulmonary disease: a systematic review. Chest 2018; 153: 888-921. doi:10.1016/j.chest.2018.01.024

14 Pasipanodya JG, Ogbonna D, Ferro BE, et al. Systematic review and meta-analyses of the effect of chemotherapy on pulmonary Mycobacterium abscessus outcomes and disease recurrence. Antimicrob Agents Chemother 2017; 61: e01206. doi:10.1128/AAC.01206-17

15 Diel R, Ringshausen F, Richter E, et al. Microbiological and clinical outcomes of treating non-Mycobacterium avium complex nontuberculous mycobacterial pulmonary disease: a systematic review and meta-analysis. Chest 2017; 152: 120-142. doi:10.1016/j.chest.2017.04.166

16 Wallace RJ Jr, Brown-Elliott BA, McNulty S, et al. Macrolide/azalide therapy for nodular/bronchiectatic Mycobacterium avium complex lung disease. Chest 2014; 146: 276-282. doi:10.1378/chest.13-2538

17 Jhun BW, Kim SY, Moon SM, et al. Development of macrolide resistance and reinfection in refractory Mycobacterium avium complex lung disease. Am J Respir Crit Care Med 2018; 198: 1322-1330. doi:10.1164/ rccm.201802-03210C

18 Koh WJ, Jeong BH, Kim SY, et al. Mycobacterial characteristics and treatment outcomes in Mycobacterium abscessus lung disease. Clin Infect Dis 2017; 64: 309-316. doi:10.1093/cid/ciw724

19 Andréjak C, Thomsen VØ, Johansen IS, et al. Nontuberculous pulmonary mycobacteriosis in Denmark: incidence and prognostic factors. Am J Respir Crit Care Med 2010; 181: 514-521. doi:10.1164/rccm.200905-0778OC

20 Hayashi M, Takayanagi N, Kanauchi T, et al. Prognostic factors of 634 HIV-negative patients with Mycobacterium avium complex lung disease. Am J Respir Crit Care Med 2012; 185: 575-583. doi:10.1164/rccm.201107-1203OC

21 Mirsaeidi M, Machado RF, Garcia JG, et al. Nontuberculous mycobacterial disease mortality in the United States, 1999-2010: a population-based comparative study. PLoS One 2014; 9: e91879. doi:10.1371/journal.pone.0091879

22 Fleshner M, Olivier KN, Shaw PA, et al. Mortality among patients with pulmonary non-tuberculous mycobacteria disease. Int J Tuberc Lung Dis 2016; 20: 582-587. doi:10.5588/ijtld.15.0807

23 Jeong BH, Jeon K, Park HY, et al. Intermittent antibiotic therapy for nodular bronchiectatic Mycobacterium avium complex lung disease. Am J Respir Crit Care Med 2015; 191: 96-103. doi:10.1164/rccm.201408-1545OC

24 Koh WJ, Jeong BH, Jeon $\mathrm{K}$, et al. Oral macrolide therapy following short-term combination antibiotic treatment of Mycobacterium massiliense lung disease. Chest 2016; 150: 1211-1221. doi:10.1016/j.chest.2016.05.003 

caused by nontuberculous mycobacteria. Am J Respir Crit Care Med 1997; 156: S1-25. doi:10.1164/ajrccm.156.1. ed08-97

26 Yang B, Jhun BW, Moon SM, et al. A clofazimine-containing regimen for the treatment of Mycobacterium abscessus lung disease. Antimicrob Agents Chemother 2017; 61: e02052.

27 Jhun BW, Yang B, Moon SM, et al. Amikacin inhalation as salvage therapy for refractory nontuberculous mycobacterial lung disease. Antimicrob Agents Chemother 2018; 62: e00011.

28 Hastie T, Tibshirani R, Friedman J. The Elements of Statistical Learning, Data Mining, Inference, and Prediction. 2nd edn. New York, Springer, 2009; pp. 43-94.

29 Yeung MW, Khoo E, Brode SK, et al. Health-related quality of life, comorbidities and mortality in pulmonary nontuberculous mycobacterial infections: a systematic review. Respirology 2016; 21: 1015-1025. doi:10.1111/ resp. 12767

30 Diel R, Lipman M, Hoefsloot W. High mortality in patients with Mycobacterium avium complex lung disease: a systematic review. BMC Infect Dis 2018; 18: 206. doi:10.1186/s12879-018-3113-X

31 Morimoto K, Iwai K, Uchimura K, et al. A steady increase in nontuberculous mycobacteriosis mortality and estimated prevalence in Japan. Ann Am Thorac Soc 2014; 11: 1-8. doi:10.1513/AnnalsATS.201303-067OC

32 Gommans EP, Even P, Linssen CF, et al. Risk factors for mortality in patients with pulmonary infections with non-tuberculous mycobacteria: a retrospective cohort study. Respir Med 2015; 109: 137-145. doi:10.1016/ j.rmed.2014.10.013

33 Novosad SA, Henkle E, Schafer S, et al. Mortality after respiratory isolation of nontuberculous mycobacteria. A comparison of patients who did and did not meet disease criteria. Ann Am Thorac Soc 2017; 14: 1112-1119.

34 Vinnard C, Longworth S, Mezochow A, et al. Deaths related to nontuberculous mycobacterial infections in the United States, 1999-2014. Ann Am Thorac Soc 2016; 13: 1951-1955. doi:10.1513/AnnalsATS.201606-474BC

35 Marras TK, Campitelli MA, Lu H, et al. Pulmonary nontuberculous mycobacteria-associated deaths, Ontario, Canada, 2001-2013. Emerging Infect Dis 2017; 23: 468-476. doi:10.3201/eid2303.161927

36 Koh WJ, Jeong BH, Jeon K, et al. Clinical significance of the differentiation between Mycobacterium avium and Mycobacterium intracellulare in $M$ avium complex lung disease. Chest 2012; 142: 1482-1488. doi:10.1378/ chest.12-0494

37 Koh WJ, Jeon K, Lee NY, et al. Clinical significance of differentiation of Mycobacterium massiliense from Mycobacterium abscessus. Am J Respir Crit Care Med 2011; 183: 405-410. doi:10.1164/rccm.201003-0395OC

38 Morimoto K, Nakagawa T, Asami T, et al. Clinico-microbiological analysis of 121 patients with pulmonary Mycobacteroides abscessus complex disease in Japan - an NTM-JRC study with RIT. Respir Med 2018; 145: 14-20. doi:10.1016/j.rmed.2018.10.012

39 Aksamit TR. Mycobacterium avium complex pulmonary disease in patients with pre-existing lung disease. Clin Chest Med 2002; 23: 643-653. doi:10.1016/S0272-5231(02)00022-9

40 Kim TS, Koh WJ, Han J, et al. Hypothesis on the evolution of cavitary lesions in nontuberculous mycobacterial pulmonary infection: thin-section CT and histopathologic correlation. AJR Am J Roentgenol 2005; 184: 1247-1252. doi:10.2214/ajr.184.4.01841247 